\title{
THE EQUATION OF PRESCRIBED RICCI CURVATURE
}

\author{
BY DENNIS M. DeTURCK ${ }^{1}$
}

Introduction. In [5], J. Milnor cited "understanding the Ricci tensor $R_{i k}=\Sigma g^{j l} R_{i j k l}$ " as a fundamental problem of present-day mathematics. A basic issue, then, is to determine which symmetric covariant tensors of rank two can be Ricci tensors of Riemannian metrics. The definition of Ricci curvature casts the problem of finding a metric $g$ which realizes a given Ricci curvature $R$ as one of solving a system of nonlinear second-order partial differential equations for $g$. We write these equations as

$$
\operatorname{Ricc}(g)=R \text {. }
$$

We note that there are the same number of equations as unknowns because $g$ and $R$ are both symmetric $n \times n$ matrices. However, there is a complication since any solution of $\operatorname{Ricc}(g)=R$ must also satisfy the Bianchi identity

$$
\operatorname{Bian}(g, R) \equiv g^{a b}\left(R_{a m ; b}-1 / 2 R_{a b ; m}\right)=0 .
$$

These conditions contain the unknown metric $g$ and its first derivatives; note that the covariant derivatives of $R$ that appear involve $g$ via its connection.

Ultimately, one would like global results about existence, uniqueness and regularity - including topological obstructions - of metrics with prescribed Ricci tensors on manifolds. The first step, though, is to determine when one can solve the equation $\operatorname{Ricc}(g)=R$ locally, say in a neighborhood of a point $x_{0}$ in $\mathbf{R}^{n}$. This local problem is already nontrivial, even in the analytic case. We will exhibit examples showing that one can not always locally solve the equation, and we also will discuss when one can prove local solvability. Further results and details will appear in [1]. In what follows, we always assume the dimension, $n$, is at least 3.

1. The Bianchi identity. It is clear that a necessary condition for existence of a metric solving $\operatorname{Ricc}(g)=R$ is the existence of metrics solving the first-order (in $g$ ) equation $\operatorname{Bian}(g, R)=0$, so we concentrate our initial efforts here. We begin with the following nonexistence result.

EXAmple 1.1. $R=\operatorname{diag}\left(x^{1}, \pm 1, \pm 1, \ldots, \pm 1\right)$ is not $\operatorname{Ricc}(g)$ for any

Received by the editors February 4, 1980.

1980 Mathematics Subject Classification. Primary 35A07, 53B20; Secondary 35J60, $35 \mathrm{~N} 99,47 \mathrm{H} 15,53 \mathrm{B3} 0,83 \mathrm{COS}$.

1 Supported in part by National Science Foundation Grant No. MCS77-01267.

These results comprise most of the author's Ph.D. thesis. Special thanks are due Jerry Kazdan and others at the University of Pennsylvania. 
Riemannian metric $g$ in a neighborhood of 0 , because there is no metric $g$ satisfying the Bianchi identity there. In fact, $\operatorname{Bian}(g, R)=0$ implies $g^{11}=0$, which is impossible for a positive definite metric. We remark that we can construct a tensor $\boldsymbol{R}_{i j}$, zero at the origin, that is not the Ricci tensor of a metric with any signature (including a Lorentz metric).

This prompts us to consider solving the Bianchi identity for metrics $g$. We fix a "Ricci candidate" $R$ throughout the discussion. First, we note that $\operatorname{Bian}(g, R)=-\operatorname{div}(G R)$, where div is the divergence operator on symmetric tensors, i.e. if $h$ is such a tensor, $\operatorname{div}(h)=-g^{a b} h_{i a ; b}$, and $G$ is the "gravitation" operator, $G h=h_{i j}-1 / 2 g_{i j}\left(g^{a b} h_{a b}\right)$. Note that $G(\operatorname{Ricc}(g))$ is the stress-energy tensor in Einstein's theory of gravitation. The linearization of the Bianchi operator about a particular metric $g$ in the direction of the symmetric tensor $h$ is

$$
\left.\operatorname{Bian}^{\prime}(g, R) h \equiv \frac{d}{d t}\right|_{t=0} \operatorname{Bian}(g+t h, R)=R_{m}^{a}(\operatorname{div}(G h))_{a}+T_{m}^{a b} h_{a b}
$$

where $\boldsymbol{T}$ does not involve $h$. Note that this system (as a differential equation in $h$ ) is underdetermined, being $n$ equations in $1 / 2 n(n+1)$ unknowns. If $R$ is nonsingular, then this operator is underdetermined elliptic. A slight generalization of a theorem of Malgrange ([6, pp. 15-16] or [4, pp. 129-134]) then yields the existence of a submanifold (of the Banach manifold of Riemannian metrics) of solutions to $\operatorname{Bian}(g, R)=0$. To solve $\operatorname{Ricc}(g)=R$ it is natural to restrict our attention to this submanifold.

2. The main result. If the equation $\operatorname{Ricc}(g)=R$ were elliptic, then one could obtain local solvability using Malgrange's theorem as above. But it is not elliptic, as one can verify from its linearization

$$
\operatorname{Ricc}^{\prime}(g) h=1 / 2 \Delta_{L} h-\operatorname{div}^{*}(\operatorname{div}(G h))
$$

where $\Delta_{L}$ is the (Lichnerowicz) Laplacian of $g$, i.e.

$$
\Delta_{L} h=-h_{i j ; a}^{a}+\text { lower order terms }
$$

and div* is the formal (or $L^{2}$ ) adjoint of div in the space of symmetric tensors, i.e.

$$
\operatorname{div} * v=12\left(v_{i ; j}+v_{j ; i}\right)
$$

for 1 -forms $v$. We note that $\operatorname{Ricc}^{\prime}(g)$ is degenerate in the sense that every direction is characteristic for this operator. However, by using the Bianchi identity, one can obtain an elliptic system at the expense of making it overdetermined. In more detail, comparison of (1) and (2) shows that the system

$$
\operatorname{Ricc}(g)+\operatorname{div}^{*}\left(R^{-1} \operatorname{Bian}(g, R)\right)=R
$$


is elliptic. Moreover the system obtained by combining (3) and

$$
\operatorname{div} *\left(R^{-1} \operatorname{Bian}(g, R)\right)=0
$$

is, although overdetermined (twice as many equations as unknowns), equivalent to the original system $\operatorname{Ricc}(g)=R$.

THEOREM 2.1. If $R$ is a $C^{\infty}$ tensor field in a neighborhood of $x_{0}$ and if $R^{-1}\left(x_{0}\right)$ exists, then there exists a $C^{\infty}$ Riemannian metric $g$ such that $\operatorname{Ricc}(g)=$ $R$ in a neighborhood of $x_{0}$.

The proof of this theorem is the heart of the entire work. It involves a new version of the implicit function theorem using Newton iterations on (3). After each iteration we project the approximation onto the submanifold of solutions of (4) found in the previous section. Rapid convergence of Newton's method compensates for the perturbations in a way that is related to the procedure of the Nash-Moser implicit function theorem.

3. The analytic case. Although the analytic case is included in Theorem $\mathbf{2 . 1}$, it is enlightening to present another proof that yields analytic metrics of arbitrary prescribed signature with nonsingular analytic Ricci tensors.

THEOREM 3.1. Let $R$ be analytic in a neighborhood of $x_{0}$ and suppose $R^{-1}\left(x_{0}\right)$ exists. Then there exists an analytic metric $g$ (of any desired signature) such that $\operatorname{Ricc}(g)=R$ in a neighborhood of $x_{0}$.

This uses Cartan-Kähler theory in the version given by Malgrange in the appendix of [4]. The main idea is that the original system $\operatorname{Ricc}(g)=R$ does not possess "strongly prolongable" solutions, whereas the equivalent system (3), (4) does. Malgrange's theorem then guarantees existence.

We turn to the gravitational operator $G$ defined in $\S 1$, and note that it is an isomorphism. Further, we note that, given any matrix $S_{i j}$ different from zero, we can find a metric $g$ so that $G^{-1} S$ is nonsingular (recall that the definition of $G$ involves $g$ ). Using these facts we can prove

\section{COROLlaRY 3.2. Einstein's gravitational equation}

$$
G(\operatorname{Ricc}(g))=\operatorname{Ricc}(g)-1 / 2 g(\operatorname{trace} \operatorname{Ricc}(g))=S_{a b}
$$

is locally solvable for $g$, provided $S$ is analytic and $S\left(x_{0}\right) \neq 0$.

Like Theorem 3.1, the corollary is independent of the signature of $g$. The restriction to nonzero stress-energy tensors $S$ is crucial as the following example illustrates.

Example 3.3. Let $s=\Sigma_{i=1}^{n} x_{i}$. Then $S=\operatorname{diag}(s, s, \ldots, s)$ is not the stress-energy tensor of any metric of any signature in a neighborhood of 0 . This follows from the fact that $\operatorname{div}(S)=0$ necessarily, by the Bianchi identity. 
4. Remarks. (1) As will be clarified in [1], the nonellipticity of the equation $\operatorname{Ricc}(g)=R$ is actually due to the invariance of the equation under the action of the group of diffeomorphisms. This invariance yields the Bianchi identity, and motivates our analysis of $\S \S 1,2$.

(2) Although the map from metrics to Ricci tensors has proved to be not locally surjective, the map from affine connections to Ricci tensors is locally surjective, as shown by Gasqui [3]. We can strengthen his results to obtain regular connections (i.e. more differentiable than $R$ ) if $R$ is finitely differentiable.

(3) As might be expected, the two-dimensional case is simpler. There, we have the following.

Proposition 4.1. A necessary and sufficient condition that $R$ is locally the Ricci tensor of a Riemannian metric (when $n=2)$ is that $R_{i j}=\sigma \gamma_{i j}$, where $\sigma$ is a scalar function and $\gamma_{i j}$ is positive definite. On a compact 2-manifold $M, a$ tensor of the above form is the Ricci tensor of a metric on $M$ if and only if

$$
\int_{M} \sigma d V_{\gamma}=2 \pi \chi(M) \text {. }
$$

(4) Some regularity results relating to this work, as well as special results for Einstein manifolds will appear in [2].

\section{REFERENCES}

1. D. DeTurck, Existence of metrics with prescribed Ricci curvature: Local theory (to appear).

2. D. DeTurck and J. Kazdan, Some regularity Theorems in Riemannian geometry (to appear).

3. J. Gasqui, Connexions à courbure de Ricci donnée, Math Z. 168 (1979), 167-179.

4. B. Malgrange, Equations de Lie. II, J. Differential Geometry 7 (1972), 117-141.

5. J. Milnor, Problems of present-day mathematics ( $\S$ XV. Differential Geometry), Proc. Sympos. Pure Math., vol. 28 (Mathematical Developments Arising from Hilbert Problems), Amer. Math. Soc., Providence, R. I., 1973, pp. 54-57.

6. L. Nirenberg, Lectures on linear partial differential equations, CBMS Regional Conf. Ser. in Math., no. 17, Amer. Math. Soc., Providence, R. I., 1973.

DEPARTMENT OF MATHEMATICS, UNIVERSITY OF PENNSYLVANIA, PHILADELPHIA, PENNSYLVANIA 19104 